Original

\title{
Tratamiento conservador del cáncer renal mediante HIFU. Técnica, indicaciones y resultados
}

\author{
Carlos Hernández Fernández, Enrique Lledó García, David Subirá Ríos, Gonzalo Bueno Chomón \\ Servicio de Urología. Hospital General Universitario Gregorio Marañón. Madrid, España
}

\section{Resumen}

En este artículo se revisan los mecanismos de acción de los ultrasonidos de alta intensidad HIFU, así como los trabajos tanto experimentales como clínicos en relación con el tratamiento de los tumores renales.

Aunque hasta la actualidad la mayor parte de la experiencia oncológica en Urología con HIFU tiene relación con el cáncer de próstata, son cada vez más los trabajos que avalan su eficacia y seguridad en la destrucción de tumores renales, completando, junto con la crioterapia y la radiofrecuencia, el espectro de la cirugía mínimamente invasiva en el cáncer renal, tratamientos que pretenden disminuir la morbilidad operatoria alcanzando similares tasas de control oncológico.

Es aún pronto para recomendar esta técnica en la práctica clínica diaria, ya que conocemos su seguridad y pocos efectos secundarios, pero es preciso que se completen muchos de los trabajos, en marcha en la actualidad, que confirmen su eficacia oncológica a medio y largo plazo.

Palabras clave: Ultrasonidos de alta intensidad (HIFU). Cáncer renal. Cirugía mínimamente invasiva.

\section{Conservative treatment of renal cancer using HIFU. Procedure, indications, and results}

\section{Abstract}

This article reviews the mechanisms of action of high-intensity focused ultrasound (HIFU), as well as both experimental and clinical work related to renal tumor treatment.

While most currently available experience in urological tumors with HIFU has been obtained with prostate cancer, an increasing number of studies support the efficacy and safety of this procedure for renal tumor destruction. HIFU completes, with cryotherapy and radiofrequency, the spectrum of minimally invasive surgery in renal cancer, intended to decrease surgical morbidity while achieving similar oncological control rates.

It is still early to recommend this procedure for daily clinical practice because, while its safety and few side effects are known, many ongoing studies intended to confirm its mid- and long-term oncological efficacy should be completed.

Keywords: High-intensity focused ultrasound (HIFU). Renal cancer. Minimally invasive surgery.

$\mathrm{E}$ manejo de los tumores renales menores de 3 $4 \mathrm{~cm}$ ha sufrido un cambio importante en la última década. Está científicamente demostrado que la nefrectomía parcial consigue unas tasas de curación similares a la nefrectomía radical ${ }^{1,2}$ y que además puede hacerse, en un porcentaje alto de casos, mediante cirugía laparoscópica, asumiendo una tasa del 15-20\% de complicaciones ${ }^{3-5}$.

Por otro lado es cada vez más frecuente el diagnóstico precoz de los tumores renales y, por tanto, el porcentaje de tumores pequeños está aumentando con claridad en la última década, y sin embargo la información que nos dan las técnicas de imagen no permiten predecir su potencial agresividad.
En un estudio retrospectivo realizado por Remzi ${ }^{6}$, donde se analizan una serie de 543 tumores de pequeño tamaño, se detectó que un $23 \%$ eran oncológicamente agresivos, mientras que el 14,7\% fueron etiquetados como benignos.

Es en esta encrucijada donde surge, cada vez con más fuerza, el concepto de técnicas de ablación tisular mediante distintas formas de energía y dentro de ellas, junto con la crioterapia y la radiofrecuencia, es donde se engloban los ultrasonidos de alta intensidad "HIFU".

Todas ellas pretenden alcanzar objetivos similares: control oncológico parecido al resto de alternativas más invasivas, una tasa mínima de complicaciones y la posibilidad de poderse repetir en el futuro en el caso de ser necesario. 


\section{MECANISMO DE ACCIÓN}

Los ultrasonidos de alta intensidad se nutren de los mismos principios físicos que los ultrasonidos, a los que tan acostumbrados estamos los urólogos. Esta energía, generada en un foco, puede propagarse a través de los tejidos, sin dañarlos, hasta un punto focal donde se produce un incremento de la temperatura, causando la necrosis tisular, sin que se afecten los tejidos adyacente.

Este fenómeno es conocido como HIFU, ablación por ultrasonidos, cirugía focal ultrasónica FUS y piroterapia.

La ecografía convencional utiliza frecuencias de entre 1-20 MHz y con frecuencias similares los ultrasonidos son empleados también en la industria para limpieza de plásticos, purificación de aguas, etc.

Con frecuencias más altas estas ondas producen necrosis, apoptosis celular y lisis de las células.

La parada de la actividad celular se produce por encima de los $43^{\circ}$, siempre que dicha temperatura se mantenga no menos de $60^{\circ}$, en este principio se basa la llamada hipertermia, sin embargo durante el tratamiento con HIFU se consiguen temperaturas por encima de los $82^{\circ} \mathrm{C}^{7}$ y en consecuencia la destrucción celular.

También aparece un fenómeno de cavitación que es más complejo e impredecible; los ultrasonidos producen vibración de los tejidos y su estructura sufre fenómenos de compresión y descompresión brusca, pudiéndose formar burbujas de gas que condicionan un mayor aumento de la temperatura y una mayor necrosis.

La temperatura necesaria para producir necrosis tisular es un fenómeno tiempo-dependiente, siendo suficiente alcanzar $60^{\circ}$ durante 1 segundo para conseguirlo.

Sin embargo, la apoptosis, que también producen los ultrasonidos, puede ser un efecto que se alcance con menor temperatura y de forma más lenta, por lo que podemos tener efectos deletéreos en un área mayor que el directamente expuesto al punto focal.

Por tanto hay que tener en cuenta al aplicar esta tecnología que hay un efecto directo y bien controlado por la elevación de la temperatura y dos peor conocidos y que intervendrán también en la destrucción de los tejidos, como son la cavitación y la apoptosis $^{8,9}$.

Se necesita también, para alcanzar los efectos deseados de destrucción tisular una ventana acús- tica que permita el paso de los ultrasonidos, por ejemplo, la vejiga es una magnífica ventana acústica. Sin embargo los huesos, el gas intestinal y el aire interfieren con la transmisión de energía y pueden suponer una dificultad añadida a a su utilización en distintas patologías.

\section{HISTORIA DEL HIFU}

Los primeras experiencias con animales datan de la década de los 50, cuando William Fry consigue lesionar distintas zonas del cerebro de gatos y monos ${ }^{10,11}$; a partir de entonces se suceden los trabajos que demuestran sus posibilidades en el tratamiento de la enfermedad de Parkinson ${ }^{12,13}$

Las investigaciones continuaron en el campo de la neurocirugía, pero debido a las limitaciones de la técnica se progresó relativamente poco en las décadas posteriores.

Ya 1956 Burov sugirió la posibilidad de tratar el cáncer mediante HIFU ${ }^{14}$ pero no es hasta la década de los 80, cuando se desarrollan las técnicas extracorpóreas para la fragmentación de los cálculos renales, que se reinicia el interés por la tecnología de los ultrasonidos de alta intensidad y su posible aplicación a la clínica ${ }^{15}$.

En urología los primeros trabajos se refieren al tratamiento de la patología prostática benigna, conociéndose ya desde 1993 los efectos de los ultrasonidos de alta intensidad en esta glándula ${ }^{16}$, aunque, por entonces, los efectos secundarios eran frecuentes, Vallancien describe un 10\% de quemaduras en la piel ${ }^{17}$, y Koehrman con una tecnología más moderna un $4 \%{ }^{18}$.

También en la década de los 90 aparecen los primeros trabajos en cáncer prostático ${ }^{19,20}$.

El tumor vesical superficial también se ha considerado candidato a ser tratado con HIFU aunque los primeros trabajos la pasada década no han tenido continuación posterior ${ }^{21}$.

Los tumores hepáticos han sido también una de las dianas terapéuticas con ultrasonidos, tanto primarios como metastásicos y desde los primeros trabajos en experimentación animal hasta la actualidad son varios los centros en el mundo con experiencia contrastada, en ocasiones combinándose con embolización arterial del tumor ${ }^{22,23}$, siendo más frecuente su utilización en China que en Occidente.

En la actualidad existen ya experiencias preliminares en el tratamiento de tumores óseos, de mama, útero e incluso pancreáticos ${ }^{24-26}$ y de forma aún tes- 
timonial existen experiencias con esta tecnología, a nivel experimental, en realización de vasectomías, control de hemorragias o en el tratamiento del dolor 27,28 .

\section{ABLACIÓN POR HIFU DE TUMORES RENALES}

La mayor parte de las técnicas ablativas en el cáncer de riñón de pequeño tamaño ${ }^{29}$ aparece la destrucción extracorpórea mediante HIFU.

Los primeros trabajos en destrucción de tejido renal de forma extracorpórea aparecen en 1992 utilizando una tecnología derivada de los litotritores piezoeléctricos ${ }^{17}$, mejorándose la tecnología posteriomente y demostrándose su viabilidad en trabajos realizados en animales ${ }^{30,31}$.

Se demostró que utilizando bajas intensidades no existía daño tisular, mientras que si dicha intensidad era mayor de $300 \mathrm{~W} / \mathrm{cm}^{2}$ aparecían lesiones térmicas, llegando a la necrosis coagulativa cuando se alcanzan temperaturas por encima de $\operatorname{los} 56{ }^{\circ} \mathrm{C}$ y se mantienen más de $1 \mathrm{seg}$.

Una vez conocidos sus efectos en este órgano, demostrándose que existe una destrucción tisular y que puede ser evaluada con técnicas de imagen (ecografía, TAC, RMN), se iniciaron las primeras experiencias clínicas. Susani et al. ${ }^{16}$ producen lesiones necróticas en tumores renales previamente a la realización de una nefrectomía, e incluso se realizan tratamientos con intención paliativa en el seno de tumores renales muy evolucionados ${ }^{32}$.

En 2002 Kohrmann $^{33}$ publica el primer caso de un paciente diagnosticado de carcinoma renal múltiple y tratado con ultrasonidos de alta intensidad con intención curativa, consiguiendo la destrucción de dos de los tumores localizados en el polo inferior y no encontrando modificaciones significativas en el situado en el polo superior, posiblemente debido a la mala transmisión de la energía a través de la parrilla costal.

El tratamiento de una serie de 30 pacientes con tumores hepáticos o renales y tratados con HIFU es comunicada en 2005 por Illing ${ }^{34}$ haciendo hincapié en que la técnica tiene dos importantes limitaciones: el tiempo del tratamiento, que es aproximadamente de dos horas por cada $2-3 \mathrm{~cm}$ de tejido tratado y la imposibilidad de utilizarlo cuando el tumor está cerca del intestino. Junto a que las costillas impiden una correcta trasmisión de los ultrasonidos y limitan su utilización en los tumores del polo superior.
A partir de entonces son todavía pocas las publicaciones sobre esta alternativa de tratamiento ${ }^{35}$, sin embargo es de destacar la posibilidad actual de utilizar HIFU a través de un abordaje laparoscópico, como lo demuestra el grupo de Marberger tratando 10 tumores renales y minimizando, de esta forma, los inconvenientes de la vía extracorpórea. De todos los tumores tratados 7 fueron extirpados inmediatamente después su ablación para estudio anatomo-patológico, encontrándose en 4 una destrucción completa del tumor y en 3 pequeñas áreas de tejido viable ${ }^{35}$.

La mayoría de las publicaciones destacan la buena tolerancia del procedimiento y la escasez de efectos secundarios.

\section{CONCLUSIONES}

Las técnicas de ablación tisular como tratamiento del cáncer de riñón de pequeño tamaño $(<4 \mathrm{~cm})$ puden cambiar el enfoque terapéutico de esta prolongación.

Los ultrasonidos de alta intensidad HIFU parecen una tecnología atractiva, tanto por vía extracorpórea como laparoscópica.

Se necesita una mayor casuística y un seguimiento a más largo plazo para que pueda ser considerado como uno de los tratamientos alternativos a la nefrectomía parcial bien abierta o laparoscópica.

\section{REFERENCIAS}

1. Herr HW. Partial nephrectomy for unilateral renal carcinoma and a normal contralateral kidney. 10-year followup. J.Urol. 1999;161(1):33-34.

2. Hafez KS, Fergany AF, Novick,AC. Nephron sparing surgery for localized renal cell carcinoma:impact of tumor size on patient survival, tumor recurrence and TNM. J.Urol. 1999;162(6):1930-1933.

3. Gill IS, Desai MM, Kaouk JH, Meraney AM, Murphy DP, Sung GT, et al.Laparoscopic partial nephrectomy for renal tumor: duplicating open surgical techniques. J.Urol. 2002;167(2 Pt 1):467-469.

4. Rosales Bordes A, Salvador Bayarri J, de Graeve N, Palou Redorta J, Villavicencio Mavrich H. Nefrectomía parcial laparoscópica transperitoneal en el tratamiento del tumor renal. Actas Urol Esp. 2006;30(5):492-500.

5. Albqami N, Janetschek G. Laparoscopic partial nephrectomy. Curr Opin Urol. 2005;15(5):306-311.

6. Remzi M, Katzenbeisser D, Waldert M, Klingler HC, Susani M, Memarsadeghi M, et al: Renal tumor size measured radiologically before surgery is an unreliable variable for predicting hisptopathological features: benign tumours are not necessarily small. BJU Int. 2007;99(5):1002-1006 .

7. Ter Haar GR, Clarke RL, Vaughan MG, Hill CR. Trackless surgery using focused ultrasound:Technique and case report. Min Inv Ther. 191;1:13-15.

8. Vykhodtseva N, McDannold N, Martin H, Bronson RT, Hynynen K. Apoptosis in ultrasound-produced threshold lesions in the rabbit brain. Ultrasound Med Biol. 2001;27(1):111-117.

9. Ashush H, Rozenszajn LA, Blass M, Barda-Saad M, Azimov D, Radnay $\mathrm{J}$ et al. Apoptosis induction of human myeloid leukemic cells by ultrasound exposure. Cancer Res. 2000;60(4):1014-1020. 
10. Fry WJ, Mosberg WH, Barnard JW, Fry FJ. Production of focal destructive lesions in the central nervous system with ultrasound. J Neurosurg. 1954;11(5):471-478.

11. FryWJ, Barnard JW, Fry FJ, Krumins RF, Brennan JF. Ultrasonic lesions in the mammalian central nervous system. Science. 1955;122(3168):517-518.

12. Ballantine HT, Bell E, Manlapaz J. Progress and problems in the neurological application of focused ultrasound. J. Neurosurg. 1960;17:858-876.

13. Lele PP. Production of deep focal lesions by focused ultrasound-current status. Ultasonics. 1967,5:105-112.

14. Kennedy JE, Ter Haar GR, Cranston D. High intensity focused ultrasound: surgery of the future?. Br J Radiol. 2003;76(909): 590-599.

15. Chaussy C, Brendel W, Schmiedt E. Extracorporeally induced destruction of kidney stones by shock waves. Lancet. 1980;2(8207): 1265-1268.

16. Susani M, Madersbacher S, Kratzik C, Vingers L, Marberger M. Morphology of tissue destruction induced by focused ultrasound. Eur Urol.1993; 23(Suppl 1):34-38.

17. Vallancien G, Chartier-Kastler E, Bataille N, Chopin D, Harouni M, Bougaran J. Focused extracorporeal pyrotherapy. Eur Urol. 1993;23(Suppl. 1)48-52.

18. Koehrmann KU, Michel MS, Fruhauf J, Volz J, Back W et al High-intensity focused ultrasound for noninvasive ablation in the kidney, prostate and uterus. J Urol 2000;163(4 Suppl): 156.

19. Gelet A, Chapelon JY, Bouvier R, Souchon R, Pangaud C, Abdelrahim AF et al. Treatment of prostate cancer with transrectal focused ultrasound : Early clinical experience. Eur Urol. 1996;29(2): 174-183.

20. Chaussy C, Thuroff S, Zimmermann R. Localized prostate cancer treated by transrectal hig-intensity focused ultrasound (HIFU) Outcome of 150 patients after 3 years. J Urol. 1999; $161: 331$.

21. Vallancien G, Harouni M, Guillonneau B, Veillon B, Bougaran J. Ablation of superficial bladder tumors with pyrotherapy. Urology. 1996;47(2):204-207.

22. Moore WE, Lopez RM, Matthews DE, Sheets PW, Etchison MR, Hurwitz AS, et al. Evaluation of high-intensity therapeutic ultrasound in the treatment of experimental hepatoma. J Pediatr Surg. 1989;24(1):30-33.

23. Wu F, Wang ZB, Chen WZ, Zou JZ, Bai J, Zhu H et al: Advanced hepatocellular carcinoma: treatment with highintensity focused ultrasound ablation combined with transcatheter arterial embolization. Radiology. 2005;235(2):659667.

24. Vaezy S, Fujimoto VY, Walker C, Martin RW, Chi EY, Crum LA. Treatment of uterine fibroid tumour in a nude mouse model using high-intensity focused ultrasound. Am.J.Obstet.Gynecol. 2000;183(1):6-11.
25. Hynynen K, Pomeroy O, Smith DN, Huber PE, McDannold NJ, Kettenbach $\mathrm{J}$, et al. MR imaging-guided focused ultrasound surgery of fibroadenomas in the breast: A feasibility study. Radiology. 2001;219(1):176-185.

26. Yuan C, Yang L, Yao C. Observation of high intensity focused ultrasound treating 40 cases of pancreatic cancer (in Chinese). Chin J Clin Hepatol. 2003;19:145-146.

27. Roberts WW, Chan DY, Fried NM, Wright EJ, Nicol T, Jarrett TW, et al. High-intensity focused ultrasound ablation of the vas deferens in a canine model. J Urol. 2002;167(6):2613-2617.

28. Dubinsky TJ, Cuevas C, Dighe MK, Kolokythas O, Hwang JH. High-intensity focused ultrasound: Current potential and oncologic applications. AJR. 2008;190(1):191-199.

29. Clayman,R.: From knife to needle to nothing. J Endourol suppl, 2000;14:63.

30. Adams JB, Moore RG, Anderson JH, Strandberg JD, Marshall FF, Davoussi LR. High-intensity focused ultrasound ablation of rabbit kidney tumors. J Endourol. 1996;10(1):71-75.

31. Visioli AG, Rivens IC, ter Haar GR et al.iißFalta título!!!. Eur J Ultrasound 1999;9:11.

32. Wu F, Wang ZB, Chen WZ, Bai J, Zhu H, Qiao TY. Preliminary experience using high intensity focused ultrasound for the treatment of patients with advanced stage renal malignancy. J.Urol. 2003;170(6 Pt 1):2237-2240.

33. Köhrmann KU, Michel MS, Gaa J, Marlinghaus E, Alken P. High intensity ultrasound as noninvasive therapy for multilocal renal cell carcinoma: case study and review of the literature. J.Urol. 2002;167(6):2397-2403.

34. Illing RO, Kennedy JE, Wu F, ter Haar GR, Protheroe AS, Friend PJ, et al. The safety and feasibility of extracorporeal high-intensity focused ultrasound (HIFU) for the treatment of liver and kidney tumours in a Western population. Br J Cancer. 2005;93(8):890-898.

35. Häcker A, Michel MS, Marlinghaus E, Köhrmann KU, Alken P. Extracorporeally induced ablation of renal tissue by high. intensity focused ultrasound. BJU Int. 2006;97(4):779-785.

36. Klingler HC, Susani M, Seip R, Mauermann J, Sanghvi N, Marberger MJ. A novel approach to energy ablative therapy of small renal tumours: laparoscopic high-intensity focused ultrasound. Eur Urol. 2008;53(4):810-818.

Correspondencia autor: Dr. Carlos Hernández Fernández Servicio de Urología

Hospital General Universitario Gregorio Marañón

Doctor Esquerdo, 46 - 28007 Madrid

Tel.: 915868000

E-mail autor: carloshfdez@telefonica.net

Información artículo: Original

Trabajo recibido: abril 2009

Trabajo aceptado: mayo 2009 